\title{
Análisis pragmalingüístico del debate electoral peruano: El caso de Fernando Olivera y Alan García ${ }^{1}$
}

\section{Pragmalinguistic analysis of the peruvian electoral debate: The case of Fernando Olivera and Alan García}

\author{
Sindy Pérez Taype \\ Universidad Nacional Mayor de San Marcos \\ sindy.perez@unmsm.edu.pe \\ https://orcid.org/oooo-0oo1-6219-6668
}

\begin{abstract}
Resumen
En el presente estudio se analiza el debate electoral del año 2016, específicamente el enfrentamiento entre los candidatos Fernando Olivera y Alan García. Se decidió trabajar con este corpus, ya que se evidencia un contraste en las estrategias pragmalingüísticas utilizadas por ambos candidatos; por lo tanto, el objetivo principal es evidenciar cada una de estas estrategias. En la primera parte del estudio se analiza el contenido del discurso político de ambos candidatos, para ello se emplea la teoría funcional propuesta por William Benoit (2007). En función de este análisis, en la segunda parte se describen los recursos pragmalingüísticos presentes en los discursos de cada uno de ellos. Se concluye que ambos candidatos presentan diferencias en el contenido de su mensaje: mientras que el discurso de Fernando Olivera tiene una propensión denigratoria, Alan García tiene una propensión al silencio. Asimismo, los recursos pragmalingüísticos más empleados son la descortesía verbal y deixis personal.
\end{abstract}

Palabras claves: debate electoral peruano, teoría funcional, descortesía, deixis personal

\begin{abstract}
This study analyzes the electoral debate of 2016, specifically the confrontation between the candidates Fernando Olivera and Alan García. It was decided to work with this corpus, since a contrast is evident in the pragmalinguistic strategies used by both candidates; therefore, the main objective is to demonstrate each of these strategies. In the first part of the study, the content of the political discourse of both candidates is analyzed, for this the functional theory proposed by William Benoit (2007) is used. Based on this analysis, the second part describes the pragmalinguistic resources present in the speeches of each one of them. It is concluded that both candidates present differences in the content of their message: while Fernando Olivera's speech has a denigrating propensity, Alan García has a propensity for silence. Likewise, the most widely used pragmalinguistic resources are verbal discourtesy and personal deixis.
\end{abstract}

Keywords: Peruvian electoral debate, functional theory, discourtesy, personal deixis

Este artículo se deriva de la investigación de la tesis de maestría en Lingüística que se presenta en la Unidad de Posgrado de la Facultad de Letras y Ciencias Humanas de la Universidad Nacional Mayor de San Marcos. 
Análisis pragmalingüístico del debate electoral peruano: El caso de...

Sindy Pérez Taype

\section{Resumo}

Este estudo analisa o debate eleitoral de 2016, especificamente o confronto entre os candidatos Fernando Olivera e Alan García. Foi decidido trabalhar com este corpus, uma vez que mostra um contraste nas estratégias pragmalinguísticas utilizadas por ambos os candidatos; por conseguinte, o principal objectivo é destacar cada uma destas estratégias. Na primeira parte do estudo, o conteúdo do discurso político de ambos os candidatos é analisado utilizando a teoria funcional proposta por William Benoit (2007). Com base nesta análise, a segunda parte do estudo descreve os recursos pragmalinguísticos presentes nos discursos de cada candidato. Conclui-se que ambos os candidatos apresentam diferenças no conteúdo da sua mensagem: enquanto o discurso de Fernando Olivera tem uma propensão denigratória, o de Alan García tem uma propensão para o silêncio. Do mesmo modo, os recursos pragmalinguísticos mais utilizados são a descortesia verbal e os deixis pessoais.

Palavras-chave: debate eleitoral peruano, teoria funcional, descortesia, deixis pessoais.

Recibido: I8-05-202I

Aprobado: 15-06-202I

Publicado: I9-I2-202I

\section{Introducción}

El debate electoral es un acontecimiento importante que se realiza en la mayoría de los países democráticos. En él los candidatos discuten temas de interés social en un mismo escenario y es un factor importante que influye en las decisiones de los votantes. Debido a la particularidad de este acontecimiento, han surgido diversos estudios con la finalidad de describir las estrategias discursivas y el contenido del mensaje de los candidatos políticos. Una de las recientes teorías que aborda el contenido del debate electoral es la teoría planteada por William Benoit: la teoría funcional del discurso de campaña política (2007). Este autor señala que los candidatos tienden a elegir entre tres funciones aplicadas tanto a los temas como los candidatos: pueden realizar manifestaciones afirmativas, atacar al oponente o manifestar defensas (Herrero y Benoit, 2009, p.64). Sobre la base de esta teoría, se puede ahondar en las estrategias pragmalingüísticas utilizadas por los diversos candidatos políticos.

La teoría funcional ha sido empleada en diversos estudios sobre campañas y debates presidenciales como EE. UU. (Benoit y Brazeal, 2002), México (Cruz, 2018), España (Herrero y Benoit, 2009), Finlandia (Isotalus, 2011), entre otros países. Por otro lado, sobre los recursos pragmalingüísticos del debate oral, se tiene el estudio de Blas Arroyo (2000), quien analiza las principales referencias de deixis personal en el debate entre dos líderes políticos españoles realizado en 1993, y a Encarnación Pérez (2014), quien estudia la deixis y descortesía como manifestación

$384 \quad$ Lengua y Sociedad. Revista de Lingüística Teórica y Aplicada 
de las habilidades persuasivas que posee el orador como mecanismos de protección y ataque en el desarrollo del debate parlamentario.

En el Perú, se encuentra el estudio de Arrunátegui y Palomino (2021), quienes analizan las estrategias de nominación como arma política que utilizan los políticos en los medios de comunicación; por su parte, Lovón (2018) examina las estrategias léxicas y discursivas presentes el discurso antiamazónico del expresidente Alan García. Sin embargo, no existe un estudio del discurso político desde el punto de vista pragmalingüístico, por lo tanto, con esta investigación se pretende dar cuenta de los recursos empleados por los candidatos en el contexto del debate electoral.

Para lograr nuestros objetivos, el estudio se ha dividido de la siguiente forma: en primer lugar, se presenta la introducción del trabajo, donde se contextualiza el estudio, se explicitan los objetivos y justificación del mismo. Luego, se desarrolla el marco teórico donde se explica la teoría precitada y los mecanismos pragmalingüísticos como la descortesía y la deixis personal. El tercer apartado está conformado por la metodología, donde se explica el procedimiento que se siguió para realizar el estudio. En seguida, se realiza el análisis del discurso de los candidatos en cuestión; luego, se presentan las conclusiones y, finalmente, las referencias bibliográficas.

\section{Marco teórico}

\subsection{El debate electoral}

Durante la campaña electoral existe el marketing político, que contiene elementos de distinta naturaleza: los mítines, las ruedas de prensa, los discursos de anuncio, las inserciones publicitarias y los debates (Canel, 1998, p. 50), donde este último constituye un elemento importante en la contienda electoral, porque sirve como un medio para que los ciudadanos conozcan a los candidatos y conozcan también las propuestas en torno a diferentes temas de interés social; así, como lo señala Luengo (2011):

[...] provee a los votantes la oportunidad de escuchar directamente a los candidatos y conocer de cerca su posición respecto a los temas de campaña, así como sus cualidades como políticos, sin la mediación ni el filtrado ejercido habitualmente por los medios de comunicación. (p. 82) 
Análisis pragmalingüístico del debate electoral peruano: El caso de...

Sindy Pérez Taype

Es decir, esta herramienta política brinda a las personas la oportunidad de comparar directamente las cualidades y políticas de los candidatos. Otro aspecto importante de los debates es que genera mucha información mediática, despertando el interés para diferentes estudios interdisciplinarios, pues este tipo de discurso es complejo en cuanto a contenido y efecto que pueda causar.

En el Perú, de acuerdo con Kanashiro (2014, p. 68), el primer debate presidencial televisado se llevó a cabo en 1990, en el contexto de la segunda vuelta electoral, entre los candidatos Alberto Fujimori (Cambio 90) y Mario Vargas Llosa (Fredemo); ya en el año 2011 se desarrolla por primera vez el debate electoral durante la campaña de primera vuelta, tradición que se mantiene hasta la actualidad.

Hasta el momento, se han realizado nueve debates presidenciales en el Perú. A continuación, se presenta un cuadro que explicita lo mencionado.

Tabla 1. Debates electorales presidenciales televisados en el Perú

\begin{tabular}{|c|c|c|}
\hline Año & Moderador(es) & Candidatos \\
\hline 1990 & Guido Lombardi & $\begin{array}{l}\text { Alberto Fujimori Fujimori (Cambio 90) } \\
\text { Mario Vargas Llosa (Fredemo) }\end{array}$ \\
\hline 2001 & Guido Lombardi & $\begin{array}{l}\text { Alejandro Toledo Manrique (Perú Posible) } \\
\text { Alan García Pérez (APRA) }\end{array}$ \\
\hline 2006 & $\begin{array}{l}\text { Augusto Álvarez } \\
\text { Rodrich }\end{array}$ & $\begin{array}{l}\text { Alan García Pérez (APRA) } \\
\text { Ollanta Humala Tasso (UPP) }\end{array}$ \\
\hline $\begin{array}{l}2011 \\
\left(1 .^{a} \text { vuelta }\right)\end{array}$ & José María Salcedo & $\begin{array}{l}\text { Ollanta Humala Tasso (Gana Perú) } \\
\text { Alejandro Toledo Manrique (Perú Posible) } \\
\text { Keiko Fujimori Higuchi (Fuerza 2011) } \\
\text { Pedro Pablo Kuczynski (Alianza por el Gran Cambio) } \\
\text { Luis Castañeda Lossio (Solidaridad Nacional) }\end{array}$ \\
\hline $\begin{array}{l}2011 \\
\left(2 .^{a} \text { vuelta }\right)\end{array}$ & José María Salcedo & $\begin{array}{l}\text { Ollanta Humala Tasso (Gana Perú) } \\
\text { Keiko Fujimori Higuchi (Fuerza 2011) }\end{array}$ \\
\hline $\begin{array}{l}2016 \\
\left(1 .^{\mathrm{a}} \text { vuelta) }\right.\end{array}$ & $\begin{array}{l}\text { Mávila Huertas } \\
\text { José María Salcedo }\end{array}$ & $\begin{array}{l}\text { Alejandro Toledo (Perú Posible) } \\
\text { Pedro Pablo Kuczynski (Peruanos Por el Kambio) } \\
\text { Luis Fernando Olivera (Frente Esperanza) } \\
\text { Gregorio Santos (Democracia Directa) } \\
\text { Keiko Fujimori Higuchi (Frente Popular) } \\
\text { Miguel Hilario Escobar (Progresando Perú) } \\
\text { Isaac Alfredo Barnechea (Acción Popular) } \\
\text { Verónica Fanny Mendoza (Frente Amplio) } \\
\text { Alan García Pérez (Alianza Popular) } \\
\text { Ántero Flores Araoz (Partido Político Orden) }\end{array}$ \\
\hline
\end{tabular}


Análisis pragmalingüístico del debate electoral peruano: El caso de...

Sindy Pérez Taype

\begin{tabular}{|c|c|c|}
\hline Año & Moderador(es) & Candidatos \\
\hline $\begin{array}{l}2016 \\
\left(2 .^{a} \text { vuelta }\right)\end{array}$ & $\begin{array}{l}\text { Mávila Huertas } \\
\text { José María Salcedo }\end{array}$ & $\begin{array}{l}\text { Pedro Pablo Kuczynski (Peruanos Por el Kambio) } \\
\text { Keiko Fujimori Higuchi (Frente Popular) }\end{array}$ \\
\hline $\begin{array}{l}2021 \\
\left(1 .{ }^{a} \text { vuelta }\right)\end{array}$ & $\begin{array}{l}\text { Mónica Delta } \\
\text { Pedro Tenorio }\end{array}$ & $\begin{array}{l}\text { George Forsyth (Victoria Nacional) } \\
\text { Verónika Mendoza (Juntos por el Perú) } \\
\text { Alberto Beingolea (Partido Popular Cristiano) } \\
\text { Keiko Fujimori (Fuerza Popular) } \\
\text { Marco Arana (Frente Amplio) } \\
\text { César Acuña (Alianza para el Progreso) } \\
\text { Pedro Castillo (Perú Libre) } \\
\text { Daniel Urresti (Podemos Perú) } \\
\text { Andrés Alcántara (Democracia Directa) } \\
\text { José Vega (Unión por el Perú) } \\
\text { Ollanta Humala (Partido Nacionalista) } \\
\text { Hernando de Soto (Avanza País) } \\
\text { Rafael López Aliaga (Renovación Popular) } \\
\text { Yohny Lescano Ancieta (Acción Popular) } \\
\text { Julio Guzmán (Partido Morado) } \\
\text { Daniel Salaverry (Somos Perú) } \\
\text { Rafael Santos (Perú Patria Segura) } \\
\text { Ciro Gálvez (Renacimiento Unido Nacional) }\end{array}$ \\
\hline $\begin{array}{l}2021 \\
\left(2 .{ }^{a} \text { vuelta }\right)\end{array}$ & $\begin{array}{l}\text { Doris Cornejo } \\
\text { César Espinoza }\end{array}$ & $\begin{array}{l}\text { Pedro Castillo (Perú Libre) } \\
\text { Keiko Fujimori (Fuerza Popular) }\end{array}$ \\
\hline
\end{tabular}

Nota: Adaptado de Debates electorales televisados: El puritanismo político audiovisual peruano, por L. Kanashiro Nakahodo, 2014, Xxir Congreso Latinoamericano de Investigadores de la Comunicación. Elaboración propia.

\subsection{Teoría funcional}

Una de las teorías más empleadas en la investigación sobre debates electorales es la teoría funcional propuesta por William Benoit (2007). De acuerdo con esta teoría, se considera funcional el discurso político, pues el objetivo es ganar las elecciones. Este hecho lleva a los candidatos a emitir mensajes con la finalidad de hacerlos parecer mejores que sus oponentes, los cuales tienen tres opciones para el contenido discursivo que quieran expresar: 1) elogio, es decir, elogiarse de los logros o cualidades positivas de uno, 2) ataque, cuando se critica al oponente por errores o cualidades negativas y 3 ) defensa, que consiste en refutar los ataques del adversario. La teoría funcional de discurso de campaña política también sostiene que estos mensajes pueden ocurrir sobre dos temas: política y carácter. Los comentarios sobre política abordan problemas que pueden ser objeto de acciones gubernamentales o no gubernamentales y pueden abordar hechos pasados, planes futuros 
Análisis pragmalingüístico del debate electoral peruano: El caso de...

Sindy Pérez Taype

y metas generales. Los comentarios sobre carácter nos informan más sobre los candidatos mismos como personas y pueden discutir las cualidades personales (coraje, honestidad, por ejemplo), la capacidad de liderazgo de los candidatos (experiencia) y los ideales de los candidatos (valores y principios).

Además, plantea cinco axiomas que proporcionan la base de su teoría:

- La votación es un acto comparativo.

- Los candidatos deben distinguirse de los oponentes.

- Los mensajes de las campañas políticas permiten distinguir a los candidatos.

- Los candidatos establecen la preferencia a través de la aclamación, el ataque y la defensa.

- El discurso de la campaña se desarrolla sobre dos temas: política y carácter.

Para los propósitos del presente estudio, el cuarto y quinto axioma de la teoría funcional resultan interesantes. La teoría funcional señala que en las campañas electorales la política suele ser el tema más frecuente, donde además pueden abordar temas relacionados a hechos pasados, planes futuros o metas generales.

\subsection{Mecanismos pragmalingüísticos}

\subsubsection{La descortesía}

De acuerdo con Escandell (1999), es importante utilizar los medios del lenguaje necesarios para lograr una relación cordial, es decir, emplear un conjunto de estrategias conversacionales para mantener las buenas relaciones y esto se conoce como cortesía. Sin embargo, en el debate electoral no se observan estas buenas relaciones, específicamente en el encuentro de los candidatos Fernando Olivera y Alan García, pues este suceso se convirtió en un campo de batalla, donde predominó la acusación o «agresión», ese deseo de vencer al adversario que lo conduce a la necesidad de hundirlo si fuese necesario. Estas actitudes conflictivas constituyen uno de los recursos principales de la descortesía (Fernández, 2000, p.112).

En el siguiente fragmento puede observarse un claro ejemplo de ello:

Fernando Olivera: Soy un hombre de fe y usted no me es indiferente, doctor García porque usted encarna la impunidad, porque usted ha pervertido los

388 Lengua y Sociedad. Revista de Lingüística Teórica y Aplicada 
valores del Perú, porque tiene que responder ante la justicia por el enriquecimiento ilícito, por el всCI, los Mirage y el Fiscal Morgenthau, por su departamento de París, por el dólar $M U C$, por la fundación Rayons de soleil que usted creó en Ginebra con sede en Liechtenstein, por el caso Lava Jato y las coimas de Odebrecht, por las coimas del tren eléctrico $[\ldots]^{2}$.

En este caso, el candidato Fernando Olivera ataca directamente a su oponente y para ello recurre a un mecanismo descortés (argumentum ad personam). Una de las primeras teorías de la descortesía fue propuesta por Culpeper (1996), que surge como reacción a los planteamientos de Brown y Levinson (1987), quienes señalaban que el comportamiento de una persona está orientado siempre a ser cooperativo. Culpeper menciona que no siempre es así, pues a veces puede haber conflictos. Él plantea cinco estrategias de descortesía; sin embargo, existen algunas ambigüedades en su clasificación. Blas Arroyo (2011) ha encontrado cierta debilidad en su propuesta, por ejemplo, la combinación de estrategias de diferente estatus. Debido a esto y con el fin de completar algunos vacíos, este autor realiza una clasificación de las diversas estrategias de descortesía en el debate oral, las cuales son:

1. Asocia directamente al interlocutor con hechos, intenciones, valores perniciosos.

2. Dile que miente.

3. Muéstrate despectivo con el adversario.

4. Formula contrastes desventajosos para el interlocutor.

5. Acusa al interlocutor de contradictorio.

\subsubsection{Deixis personal en el debate político}

Levinson (2006) menciona que el lenguaje posee una naturaleza simbólica/ indexada. La indexicalidad implica una relación entre el enunciado y el contexto para encontrar la referencia deseada. La categoría gramatical de persona refleja directamente los diferentes roles que los individuos juegan en el evento

2 El expresidente Alan García enfrentó una serie de acusaciones, entre las que destacan los sobornos del BCCI, la compraventa de aviones Mirage, irregularidades en la ejecución del tren eléctrico, indultos a sentenciados por narcotráfico, Sobornos de Odebrecht, lavados de activos con la Fundación Rayons de Soleil, entre otros. 
Análisis pragmalingüístico del debate electoral peruano: El caso de...

Sindy Pérez Taype

comunicativo: orador, destinatario, entre otros. Cuando estos roles cambian en el curso del evento conversacional, el orador en referencia también cambia con ellos.

(1) El paradigma de la persona tradicional puede ser descrito mediante la inclusión de locutor $(\mathrm{S})$ e inclusión de destinatario (A). Primera persona $(+\mathrm{S})$, segunda persona $(+\mathrm{A},-\mathrm{S})$ y tercera persona $(-\mathrm{S},-\mathrm{A})$. El autor también señala que existe una gran complejidad respecto al pronombre de primera persona plural, pues no implica más de una persona en el rol $(+S)$; muchas lenguas discuten sobre distinguir tales grupos como: $+\mathrm{S}+\mathrm{A}$ vs $+\mathrm{S}+\mathrm{A}+\mathrm{O}$ ( $\mathrm{O}$ es Otro), frente $\mathrm{a}+\mathrm{S}+\mathrm{A}+\mathrm{O}$. (p.113).

En el contexto político, se observa el empleo de deícticos como estrategias con fines persuasivos. Los candidatos manipulan los deícticos para asumir o desligar responsabilidades o para establecer la solidaridad con los electores. Además, a través de los deícticos personales, el candidato se identifica como parte de una agrupación, incluso marca distancia con el oponente. Por ello, el lugar, el tiempo, los participantes y el contenido del discurso son fundamentales para la selección de la deixis adecuada, la cual puede ser pronombre personal, verbos flexionados, posesivos y demostrativos. En suma, el estudio de la deixis personal puede ofrecer una ventana para visualizar el pensamiento de estos personajes.

\subsubsection{El espacio del discurso del hablante}

En el debate electoral se pueden distinguir dos tipos de participantes principales. El primer tipo de participantes se refiere al yo del enunciado, que lucha en el debate contra el tú del oponente político (Blas, 2011). Este autor menciona además que el yo del discurso adopta dos facetas claramente distinguibles: el neutral yo, que responde a las necesidades del hablante en momentos específicos de la interacción; por otro lado, existe el yo presidencial, que presenta un alto contenido político. La presencia del yo presidencial distingue al hablante como el jefe que representa a un grupo político y también le da más peso como líder social y político.

El segundo participante es nosotros exclusivo, que, además, "puede adoptar diversos perfiles en función del espacio de discurso en el que se sitúan los participantes en cada caso» (Blas, 2011, p. 123). Existe, por ejemplo, el nosotros partidista, que incluye al candidato y a los miembros del partido político, nosotros inclusivo, donde se incluye al candidato más los interlocutores, entre otros. 


\subsubsection{El espacio del discurso del interlocutor}

Blas Arroyo (2011) nos habla del mundo del interlocutor, al que se refiere a las formas usted o ustedes, sus variantes pronominales y variantes verbales, la forma usted aparece generalmente cuando el hablante quiere criticar al interlocutor, es decir, para ataques y agresiones políticas. Esto hace que este pronombre aparezca de forma explícita, a comparación de los casos en los que la deixis aparece incorporada morfológicamente en el verbo, algo común en el español.

Lo mismo sucede en el caso de ustedes: se alterna su uso con usted en la misma secuencia crítica, donde el ataque trasciende al partido político del oponente. En conclusión, el ataque no solo se puede dar a nivel individual, sino también a nivel del colectivo que representa el oponente.

\section{Metodología}

El presente estudio es de enfoque cualitativo, descriptivo y se inscribe en la línea de lingüística textual con una orientación pragmalingüística. Se analiza el debate presidencial de primera vuelta realizado el o3 de abril del 2016, específicamente el encuentro entre los candidatos Fernando Olivera y Alan García. Se eligió este enfrentamiento debido al contraste que se evidencia en el empleo de los recursos pragmalingüísticos. Para analizarlo se realizó una transcripción total del debate, luego se seleccionaron los discursos de los candidatos mencionados.

Para la primera parte del análisis, de acuerdo con la propuesta de Benoit (2007), se caracterizan los textos de cada candidato según su función: propuestas, ataques o defensas. Se describe también la orientación del discurso: política o carácter. Finalmente, se identifica el subtema correspondiente: los temas de política se clasifican en hechos pasados, planes futuros y metas generales; mientras que los temas de carácter, según cualidades personales, habilidades de liderazgo e ideales.

En la segunda parte del análisis, se identifican los mecanismos pragmalingüísticos. En primer lugar, se identifican los enunciados descorteses. Una vez identificados los enunciados, se establece el contexto de su aparición y se clasifican los tipos de descortesía, de acuerdo con la clasificación de Blas Arroyo (2011) que se expuso en el marco teórico. En segundo lugar, se identifican y describen los recursos deícticos. Se realiza un contraste entre los pronombres yo/nosotros frente al tú(usted)/ ustedes, según las propuestas de Levinson (2006) y Blas Arroyo (2011). 


\section{Análisis}

\subsection{La estructura del debate}

El debate de primera vuelta realizado en abril del 2016 tuvo una duración de una hora con quince minutos, fue organizado por el Jurado Nacional de Elecciones (JNE) y fue transmitido por señal abierta a todo el Perú. Este debate contó con la participación de once candidatos a la presidencia de la República. La ubicación, el uso de la palabra y las duplas de los candidatos y candidatas se dieron de acuerdo con un sorteo previo realizado el 28 de marzo en presencia de los representantes de las organizaciones políticas y de un notario público.

El debate del 2016 estuvo constituido por cuatro bloques que serán brevemente explicados a continuación:

\section{Tabla 2. Estructura del debate presidencial del año 2016}

\begin{tabular}{|c|c|}
\hline Primer bloque & $\begin{array}{l}\text { Presentación inicial para abordar el tema general Visión del país. } \\
\text { En este bloque cada candidato contó con dos minutos para realizar } \\
\text { su presentación. }\end{array}$ \\
\hline Segundo bloque & $\begin{array}{l}\text { Este bloque estuvo conformado por duplas para intercambiar ideas } \\
\text { sobre economía, seguridad ciudadana y lucha contra la corrupción. } \\
\text { En total se formaron cinco grupos, donde cada candidato tuvo } \\
\text { un espacio de un minuto y treinta segundos para presentar sus } \\
\text { propuestas, luego esta exposición el oponente debía formular } \\
\text { una pregunta o comentar al respecto por un periodo de treinta } \\
\text { segundos y luego, quien inició la ronda tendrá derecho a la réplica } \\
\text { por un minuto más. Culminada la ronda, se inició una nueva con } \\
\text { la exposición del otro candidato o candidata que integró la dupla. }\end{array}$ \\
\hline Tercer bloque & $\begin{array}{l}\text { Los candidatos responden las preguntas de los ciudadanos. Este } \\
\text { debate motivó la participación ciudadana a través de la campaña } \\
\text { «Pregúntale a tu candidato» en los peruanos enviaron sus preguntas } \\
\text { procedentes de distintas partes del Perú. }\end{array}$ \\
\hline Cuarto bloque & $\begin{array}{l}\text { Cada candidato emitió su mensaje final por un periodo de un } \\
\text { minuto y treinta segundos. }\end{array}$ \\
\hline
\end{tabular}

Este debate contó también con la presencia de dos moderadores, quienes se encargaron de brindar las indicaciones y controlar los tiempos de cada candidato o candidata. 
Análisis pragmalingüístico del debate electoral peruano: El caso de...

Sindy Pérez Taype

\subsection{El contenido del debate: ataques, defensas y propuestas}

En el siguiente fragmento se evidencia el ataque por parte del candidato Fernando Olivera hacia su oponente. En efecto, en (1) se puede observar al representante de Frente Esperanza reprochar a su oponente, tocando diversos hechos sucedidos en su primer mandato.

(1) Fernando Olivera: Soy un hombre de fe y usted no me es indiferente, doctor García porque usted encarna la impunidad, porque usted ha pervertido los valores del Perú, porque tiene que responder ante la justicia por el enriquecimiento ilícito, por el BCCI, los Mirage y el Fiscal Morgenthau, por su departamento de París, por el dólar MUC, por la fundación Rayons de soleil que usted creó en Ginebra con sede en Liechtenstein, por el caso Lava Jato y las coimas de Odebrecht, por las coimas del tren eléctrico, Sergio I. y las coimas en el Gran caimán, por los crímenes como la masacre en los penales, el comando Rodrigo Franco, las ofensas a las comunidades nativas y el Baguazo, llamándolos ciudadanos nativos de segunda clase, por la persecución a los maestros, por las colas, por la inflación, por el 'paquetazo'.

Este fragmento corresponde al bloque del debate donde los candidatos debían argumentar sobre economía, seguridad y corrupción; sin embargo, Fernando Olivera optó por el ataque, señalando una serie de acusaciones que sucedieron cuando su oponente, Alan García, era presidente del Perú. El ataque que realiza se da en torno a los dos ejes temáticos; es decir, ataca la personalidad del candidato, cuando menciona: usted encarna la impunidad, porque usted ha pervertido los valores del Perú; además, es posible que esté empleando el sarcasmo al llamarlo doctor García, pues se ha demostrado que Alan García no ha obtenido este grado. También hace referencia a temas políticos cuando le increpa que tiene que responder a la justicia por enriquecimiento ilícito, el caso Lava Jato, las coimas del tren eléctrico, entre otros hechos.

Los ataques que se expresan en este debate se dan tanto a las cualidades de la persona como a temas políticos. Veamos el siguiente fragmento del candidato Fernando Olivera.

(2) FO: Como siempre, como siempre, el doctor García y la corrupción se niegan a responder. Esa es la conducta de quien controla jueces y fiscales. Eso acabará el 28 de julio. Tenga la seguridad. Usted será juzgado por la nueva ley, la nueva justicia y va a ser condenado por los jurados con la participación popular que allí habrá y por eso es que nosotros aplicaremos el mayor presupuesto de 
Análisis pragmalingüístico del debate electoral peruano: El caso de...

Sindy Pérez Taype

la República al sector educación para que un pueblo que sabe no lo engaña nadie [...] Por eso la educación libera al pueblo y por eso es que usted, doctor García Pérez, nunca ha invertido en educación, más bien ha perseguido a los maestros, a los estudiantes y también tendrá que responder.

Como podemos observar, hecho que también se evidencia en (1), el candidato expresa, en primer lugar, el ataque a nivel de carácter, específicamente a cualidades personales, que en este caso se tratan de cualidades negativas: Como siempre, como siempre, el doctor García y la corrupción se niegan a responder. Esa es la conducta de quien controla jueces y fiscales, donde da a entender que Alan García es una persona que suele evadir a la justicia debido al poder político que posee. Luego, procede a realizar reclamos por no invertir en educación cuando este era presidente y la persecución que realizaba a docentes y estudiantes.

Más adelante, el candidato Fernando Olivera continúa con los ataques, como vemos en el siguiente fragmento:

(3) Fo: A ese nuevo sepa de la Amazonía, usted irá a trabajar condenado por la nueva justicia y qué cara tiene de venir a hablarnos de seguridad cuando en su gobierno se engendró la mayor inseguridad, cuando usted preparó el túnel para que se fuguen sus amigos del MRTA con Víctor Polay a la cabeza, cuando rindió homenaje a Sendero Luminoso, como ejemplo de mística y entrega. De qué seguridad nos viene a hablar, doctor Alan García, hay que tener cara dura, pero el pueblo ya no le cree.

El candidato continúa, en primer lugar, con ataques hacia la persona, con calificativos como caradura y mentiroso, y, en segundo lugar, realiza acusaciones políticas como la inseguridad y participación en la liberación de terroristas. Finalmente, observemos el siguiente fragmento que corresponde al último bloque del debate. Esta última intervención debía ser un mensaje final para la población; sin embargo, Fernando Olivera aprovechó una vez más para atacar a su oponente:

(4) Cumpliré con la deuda social al magisterio, a los postergados jubilados, reajustándoles sus pensiones, acabando la persecución a los jubilados de la 20530, incluidos militares con reajustes y haremos que también los discapacitados sean considerados y se cumpla la ley con ellos. Tenemos que cumplir y devolver los derechos conculcados a los trabajadores. Así haremos el fin de los sérvices, no como el doctor García, que prometió y no cumplió durante su gobierno $[\ldots]$ 
Como se puede observar, la temática del discurso de Fernando Olivera gira en torno a críticas y ataques hacia su adversario, Alan García. Los ataques son tanto al carácter como a temas políticos, especialmente acusaciones con relación a los gobiernos anteriores de Alan García, pues este ya fue dos veces presidente del Perú. El discurso de Fernando Olivera está claramente orientado hacia la denigración y deja de lado la presentación de sus propuestas en la mayor parte de su participación.

Ahora, respecto al discurso del candidato Alan García, lo que se esperaría luego de esta serie de ataques, es la defensa de su parte, que a menudo es acompañada de un contraataque. El siguiente fragmento muestra la respuesta de Alan García ante los ataques del candidato de Frente Esperanza.

(5) AG: Yo no recojo ofensas ni las comento, mucho menos por parte de quien ha sido capaz de llevar cartas falsificadas ante el Papa para calumniar al cardenal del Perú, Juan Luis Cipriani. Solo quisiera volver al punto fundamental: necesitamos hacer justicia a sectores importantes como los taxistas y mototaxistas brindándoles jubilación y seguro de salud con el Impuesto selectivo al consumo.

Si bien el candidato no se defiende de las acusaciones, sí realiza un contraataque al expresar: "Yo no recojo ofensas ni las comento, mucho menos por parte de quien ha sido capaz de llevar cartas falsificadas ante el Papa para calumniar al cardenal del Perú, Juan Luis Cipriani». Se observa que Alan García trata de minimizar lo mencionado por Fernando Olivera y para ello emplea la despersonalización, es decir, rompe el eje yo/usted y en su lugar emplea yo/tercera persona, cuando lo acusa de haber llevado cartas falsificadas. Luego de ello, desvía su mensaje hacia un eje político, donde menciona beneficios para los taxistas y mototaxistas.

Más adelante, se observa que el candidato Alan García evade por completo el ataque del candidato Fernando Olivera y dirige su discurso hacia temas políticos.

(6) AG: Toco los temas concretos que la población espera, así como el de los taxistas y mototaxistas. La titulación que es importantísima para poner un valor económico en los bancos y en apoyo al crédito y a la pequeña industria. Además, el agua potable a la cual destinaremos en cinco años 10 mil millones de soles en proyectos ya hechos que servirán a 5 millones de peruanos y 4 mil millones de soles en pistas y veredas del nuevo programa que traemos para impulsar el mayor bienestar de las familias y la salud de los niños [...]. 
Análisis pragmalingüístico del debate electoral peruano: El caso de...

Sindy Pérez Taype

En esta parte del debate, Alan García debía dar una réplica a lo mencionado anteriormente por Fernando Olivera, quien lo había acusado de generar la mayor inseguridad durante su gobierno anterior. Ante esta acusación, Alan García inicia su discurso con la frase Toco los temas concretos que la población espera [...]. De esto se podría deducir que para Alan García es insignificante lo expresado por Olivera, al punto de no merecer una respuesta directa, y toca temas relevantes, según considera el candidato, para la población. Luego, continúa con la mención de sus propuestas: la titulación, agua potable e inversiones. En el siguiente fragmento, se observa el mismo recurso:

(7) AG: Nunca he perdido el tiempo ante los insultos, he construido en el Perú y creo que el primer problema que tienen todos los peruanos ahora es el de la seguridad ciudadana y les interesa saber cómo realmente podemos responder a ese problema.

Este enunciado fue expresado después de que el candidato Fernando Olivera lo acusara de no invertir en educación mientras fue presidente. Con el silencio, Alan García quiere restar solidez a lo que expresa Olivera, con la finalidad de hacerlo invisible.

La teoría funcional también predice que los objetivos generales se utilizarán con más frecuencia para elogiar que atacar. Sin embargo, de acuerdo con el análisis se puede observar que existe una división muy marcada en los discursos de ambos candidatos. El candidato Fernando Olivera recurre al ataque, tanto de la personalidad del oponente como de temas políticos sucedidos cuando Alan García era presidente, mientras que el candidato Alan García muestra una tendencia hacia el silencio y se limita a plantear sus propuestas. Por lo tanto, se observa un contraste entre el ataque directo de Fernando Olivera y la respuesta indirecta de Alan García.

\subsection{Recursos pragmalingüísticos en el debate electoral}

Como se ha podido observar en el apartado anterior, el ataque fue la categoría más predominante en la confrontación entre Alan García y Fernando Olivera. Ahora, sobre la base de este análisis, se describirán los principales mecanismos pragmalingüísticos empleados por ambos candidatos. 
Análisis pragmalingüístico del debate electoral peruano: El caso de...

Sindy Pérez Taype

\subsubsection{La descortesía}

A continuación, se identifican las diferentes estrategias de descortesía presentes en nuestro corpus de estudio, de acuerdo con la propuesta de Blas Arroyo (2011).

\section{A. Primera estrategia: Asocia al interlocutor con hechos, intenciones, valores, etc. perniciosos.}

Esta estrategia se evidencia en el discurso del candidato Fernando Olivera, vemos en el siguiente fragmento la acusación directa al oponente, haciéndolo responsable de diversos delitos. Estas acusaciones pueden tener como destinatario al propio rival (8), o al grupo político que representa (9):

(8) Fo: Soy un hombre de fe y usted no me es indiferente, doctor García porque usted encarna la impunidad, porque usted ha pervertido los valores del Perú, porque tiene que responder ante la justicia por el enriquecimiento ilícito, por el BCCI, los Mirage y el Fiscal Morgenthau, por su departamento de París, por el dólar MUC, por la fundación Rayons de soleil que usted creó en Ginebra con sede en Liechtenstein, por el caso Lava Jato y las coimas de Odebrecht, por las coimas del tren eléctrico, Sergio I. y las coimas en el Gran caimán [...]

(9) FO: A ese Nuevo sepa del Amazonía, usted irá a trabajar condenado por la nueva justicia y qué cara tiene de venir a hablarnos de seguridad cuando en su gobierno se engendró la mayor inseguridad, cuando usted preparó el túnel para que se fuguen sus amigos del MRTA con Víctor Polay a la cabeza, cuando rindió homenaje a Sendero Luminoso, como ejemplo de mística y entrega.

(10) AG: Yo no recojo ofensas ni las comento, mucho menos por parte de quien ha sido capaz de llevar cartas falsificadas ante el Papa para calumniar al cardenal del Perú, Juan Luis Cipriani. Solo quisiera volver al punto fundamental [...]

En el fragmento (8) se observa cómo Fernando Olivera le reprocha directamente de enriquecimiento ilícito, por sobornos, por el caso Lava Jato, entre otras acusaciones. En el caso (9), el ámbito de referencia es más amplio, pues no solo hace responsable al adversario, sino también a todo su gobierno; esto incluye a congresistas y parlamentarios que hayan formado parte de dicho gobierno. En el fragmento (10), el candidato Alan García también realiza una acusación contra Fernando Olivera, con la finalidad de desacreditar todo lo antes mencionado por este. 
Análisis pragmalingüístico del debate electoral peruano: El caso de...

Sindy Pérez Taype

\section{B. Segunda estrategia: Dile que miente}

Otro tipo de estrategia planteado por Blas Arroyo es la acusación de que el adversario miente. Esta es considerada una de las mayores ofensas, pues los debates electorales son observados por una gran audiencia, audiencia de la que depende que gane o pierda las elecciones y por ello resulta perjudicial para el candidato acusarlo como mentiroso. A continuación, mostraremos ejemplos de este recurso por parte del candidato Fernando Olivera en contra de Alan García:

(11) [...] y por eso es que nosotros aplicaremos el mayor presupuesto de la República al sector educación para que un pueblo que sabe no lo engaña nadie, no lo engaña un charlatán, no lo engaña un comandante, no lo engaña una japonesa, no lo engaña nadie ${ }^{3}$.

(12) [...] de qué seguridad nos viene a hablar, doctor Alan García, hay que tener cara dura, pero el pueblo ya no le cree.

(13) [...] así haremos el fin de los sérvices, no como el doctor García, que prometió y no cumplió durante su gobierno.

Como se observa en el primer caso, el candidato repite cinco veces ese acto de mentir, donde no solo se refiere a su oponente, sino a todos los candidatos presentes en el debate usando calificativos como charlatán, comandante y japonesa. Esa repetición realza el acto de mentir y refuerza el uso de esta estrategia. En el segundo caso expresa la frase «el pueblo ya no le cree», donde da a entender que en ocasiones anteriores el candidato Alan García solía engañar a la población. Y finalmente, en (12) realiza un contraste, aludiendo que él sí cumplirá su promesa, a diferencia de Alan García que prometió y no cumplió.

\section{Tercera estrategia: Muéstrate despectivo con el adversario.}

No se han encontrado casos donde se evidencie esta estrategia.

3 El candidato utiliza el calificativo charlatán para referirse a Alan García, comandante para referirse a Ollanta Humala y japonesa para referirse a Keiko Fujimori.

398 Lengua y Sociedad. Revista de Lingüística Teórica y Aplicada 
Análisis pragmalingüístico del debate electoral peruano: El caso de...

Sindy Pérez Taype

\section{Cuarta estrategia: Formula contrastes desventajosos para el interlocutor}

Esta estrategia consiste en restarle credibilidad al oponente o acusándolo de contradictorio, como el caso (14) cuando el candidato Alan García expresa su mensaje final con el siguiente fragmento:

(14) Compatriotas, en nueve días debemos decidir el destino del Perú y estamos convencidos que (sic) sí se puede hacer un cambio fundamental en favor de los más humildes, pero no con propuestas gaseosas, de cambios constitucionales que supondrán años o renegociaciones que supondrán años.

Con esta expresión, el candidato realiza un contraste, no directamente con Fernando Olivera, sino una comparación entre sus propuestas y las planteadas por otros candidatos, aludiendo que las suyas son concretas a diferencia de otras propuestas que resultarían difícil de cumplir o que tomaría años para su ejecución.

Contraste en entre el «yo/nosotros» frente al «usted/ustedes»

A propósito de los mecanismos de descortesía, se observa un contraste entre los deícticos personales empleados en los discursos de los ambos candidatos de acuerdo con el mensaje que transmiten. A continuación, algunos ejemplos de estos casos son los siguientes:

(15) Fo: [...] usted no me es indiferente, doctor García porque usted encarna la impunidad porque usted ha pervertido los valores del Perú porque tiene que responder ante la justicia $[. .$.

(16) Fo: Usted será juzgado por la nueva ley, la nueva justicia y va a ser condenado por los jurados con la participación popular [...]

(17) FO: [...]Por eso la educación libera al pueblo y por eso es que usted, doctor García Pérez, nunca ha invertido en educación, más bien ha perseguido a los maestros, a los estudiantes y también tendrá que responder [...]

(18) Fo: A ese nuevo Sepa de la Amazonía, usted irá a trabajar condenado por la nueva justicia y qué cara tiene de venir a hablarnos de seguridad cuando en su gobierno se engendró la mayor inseguridad, cuando usted preparó el túnel para que se fuguen sus amigos del MRTA [...]

Generalmente, el pronombre «usted» suele ser empleado como un mecanismo de cortesía o respeto. Sin embargo, en este caso el candidato Fernando Olivera 
Análisis pragmalingüístico del debate electoral peruano: El caso de...

Sindy Pérez Taype

emplea frecuentemente la forma «usted» para marcar un distanciamiento con Alan García. Blas Arroyo (2011) menciona, además, que el uso más frecuente de este pronombre es para ataques y agresiones políticas; es por ello que se menciona explícitamente el sujeto a diferencia de otros casos donde se suele emplear el sujeto nulo o tácito, característico del español.

En contraste a estos casos, se presenta a continuación el empleo del deíctico «yo»:

(19) Fo: (Yo) soy un hombre de fe y usted no me es indiferente, doctor García porque usted encarna la impunidad, porque usted ha pervertido los valores del Perú $[\ldots]$

(20) Fo: Yo vengo aquí a notificarle que el 28 de julio usted no podrá volver a burlarse del país y será juzgado por la nueva justicia y será condenado [...]

(21) AG: Yo no recojo ofensas ni las comento, mucho menos por parte de quien ha sido capaz de llevar cartas falsificadas ante el Papa para calumniar al cardenal del Perú, Juan Luis Cipriani.

(22) AG: (Yo) Nunca he perdido el tiempo ante los insultos, he construido en el Perú y creo que el primer problema que tienen todos los peruanos ahora es el de la seguridad ciudadana $[\ldots]$

(23) AG: (Yo) Toco los temas concretos que la población espera, así como el de los taxistas y mototaxistas, la titulación que es importantísima para poner un valor económico en los bancos y en apoyo al crédito y a la pequeña industria $[\ldots]$

Se observa en estos casos, el yo de cada enunciado lucha contra el tú del oponente político, en los casos presentados se emplea este yo presidencial para marcar distancia con el adversario. Los candidatos polarizan el discurso, por ejemplo, cuando expresan: yo soy un hombre de fe, yo no recojo ofensas, yo toco temas concretos resaltan esa distancia entre ambos.

Por otro lado, el empleo del deíctico nosotros aparece cuando hacen referencia a los proyectos del candidato junto a su partido político, Blas Arroyo (2000) lo denomina nosotros partidario, pues este deíctico incluye tanto al candidato como a los integrantes del partido político al que representan:

$400 \quad$ Lengua y Sociedad. Revista de Lingüística Teórica y Aplicada 
Análisis pragmalingüístico del debate electoral peruano: El caso de...

Sindy Pérez Taype

(24) AG: [...] (nosotros) Necesitamos hacer justicia a sectores importantes como los taxistas y mototaxistas, brindándoles jubilación y seguro de salud con el impuesto selectivo al consumo.

(25) FO: [...] por eso es que nosotros aplicaremos el mayor presupuesto de la República al sector educación para que un pueblo que sabe no lo engaña nadie $[\ldots]$

(26) AG: [...] (nosotros) Creemos firmemente que es necesario excluir totalmente a los más peligros delincuentes en cárceles que llamaremos Los nuevos cepas en la Amazonía y exigimos al Congreso Nacional que bote la pena de muerte para los violadores de niños seguida de asesinato y para los sicarios. De esta manera garantizaremos seguridad en el país.

(27) AG: (Nosotros) Estaremos luchando también por la seguridad ciudadana.

El deíctico nosotros se emplea cuando exponen sus planes de gobierno y en estos planes se incluye tanto al candidato como a los miembros de su partido político. Se observa, por lo tanto, el compromiso tanto del candidato como de toda su asociación política para así brindar mayor confianza a los votantes.

\section{Conclusiones}

Las investigaciones revelan que las propuestas son más comunes que los ataques; sin embargo, en esta investigación se ha encontrado una división marcada entre ambos candidatos, el candidato Fernando Olivera muestra una tendencia al ataque dirigido tanto a la personalidad como a hechos políticos pasados del oponente. Por otro lado, el candidato Alan García opta por el silencio y evade las acusaciones, persiguiendo la invisibilización del rival.

Se observa que una de las estrategias más empleadas en el corpus de estudio es la descortesía, específicamente el recurso Asocia al interlocutor con hechos, intenciones, valores perniciosos. Esta estrategia fue empleada principalmente por el candidato Fernando Olivera, pues fue quien más acusaciones impugnó.

Otro mecanismo empleado en este debate es la deixis. En la investigación, se observa un uso estratégico de la deixis pronominal que es considerablemente diferente entre los dos protagonistas principales del debate. El candidato Fernando Olivera emplea frecuentemente el pronominal usted como mecanismo de distanciamiento con una connotación de encono hacia su oponente. Alan García emplea 
Análisis pragmalingüístico del debate electoral peruano: El caso de...

Sindy Pérez Taype

el yo presidencial como recurso de distanciamiento hacia su oponente; en lugar de responder las acusaciones, menciona que prefiere tocar temas relevantes. Finalmente, se observa el uso del nosotros por ambos candidatos como señal de compromiso tanto de la persona como del partido político que representan.

Finalmente, se ha podido evidenciar que los discursos políticos no son improvisados, requieren de una preparación previa donde se incluyen argumentos que el ciudadano quiere escuchar. Además, a través de su discurso, el candidato puede alardear de los propios logros y en contraste, puede realizar la deslegitimación del adversario, donde este es presentado negativamente y para ello recurre a diversos recursos pragmalingüísticos. Es necesario ahondar en este tipo de investigaciones para conocer a fondo el discurso de los candidatos políticos.

Agradecimientos

La autora agradece al lingüista Raymundo Casas Navarro por sus observaciones y revisiones para la confección del presente manuscrito. También es oportuno señalar un agradecimiento al lingüista Marco Antonio Lovón Cueva por los alcances brindados a la confección y lectura del manuscrito.

Financiamiento

La investigación se realizó sin financiamiento.

Conflicto de intereses

La autora no presenta conflicto de interés.

\section{Referencias bibliográficas}

Arrunátegui, C., y Palomino, M.-M. (2021). Los nombres en disputa: Análisis de las estrategias de nominación utilizadas por políticos en medios de comunicación peruanos durante la cobertura de dos casos de corrupción. Lengua $y$ Sociedad, 2o(1), 315-333. http://revista.letras.unmsm.edu.pe/index.php/ls/ article/view/2214

Benoit, W. (2007). Communication in political campaigns. Peter Lang.

Benoit, W. (2013). Political election debates. Lexington books

402 Lengua y Sociedad. Revista de Lingüística Teórica y Aplicada 
Análisis pragmalingüístico del debate electoral peruano: El caso de...

Sindy Pérez Taype

Blas, J. (2000). Mire usted, sr. Gonzales...Personal deixis in spanish political-electoral debate. Journal of pragmatics, 32(1), 1-27.

Blas, J. (2001). 'No digas chorradas...' La descortesía en el debate político cara a cara. Oralia, 4, 9-45.

Blas, J. (2011). Políticos en conflicto: una aproximación pragmático-discursiva al debate electoral cara a cara. Peter Lang AG.

Canel, M. (1998). Los efectos de las campañas electorales. Comunicación y Sociedad. 11(1), 47-67.

Comeca, R. (2016, 4 de abril). Debate presidencial Perú 2016. [Archivo de video]. Youtube. https://www.youtube.com/watch?v=mmuxizujvnU

Culpeper, J.(1996). Towards an anatomy ofimpoliteness. Journalofpragmatics, 25(3), 349-367.https://www.researchgate.net/publication/222497902_Towards_an_ Anatomy_of_Impoliteness

El Comercio (2019, 6 de mayo). Alan García: una vida política marcada por la polémica. El Comercio. https://elcomercio.pe/politica/alan-garcia-vida-politicamarcada-polemica-noticia-632459-noticia/?ref=ecr

Perez, E. (2014). Deixis social y (des)cortesía verbal como estrategias argumentativas. Análisis discursivo del debate político desde el enfoque sociopragmático. [Tesis para obtener el grado de doctora en Lengua española y Lingüística]. Universidad de Murcia. https://digitum.um.es/digitum/handle/10201/41867

Fernández García, F. (2000). Estrategias del diálogo. La interacción comunicativa en el discurso político-electoral. Método Ediciones.

Isotalus, P. (2011). Analyzing presidential debates. Functional theory and finnish political communication culture. Nordicom Review, 1(32), 31-43.

Herrero, J. L. y W. Benoit (2009). Análisis funcional de los debates en las elecciones generales de 2008. Zer, 14(27), 61-81.

Kanashiro, L. (2014). Debates electorales televisados: el puritanismo político audiovisual peruano. xxir Congreso Latinoamericano de Investigadores de la Comunicación.

Kanashiro, L. (2017). Debates presidenciales televisados en el Perú (1990-2011): una aproximación semiótica. Fondo Editorial Universidad de Lima.

Levinson, S. (2006). Deixis. En Horn, L. y Ward, G. The Handbook of Pragmatics. (pp. 97-121). Blackwell Publishing Ltd.

Lovón, M.-A. (2018). El «ciudadano» amazónico en el discurso político oficial. Lingüística y Literatura, 40(75), 38-61. https://doi.org/10.17533/udea.lyl. n75ao2 
Análisis pragmalingüístico del debate electoral peruano: El caso de...

Sindy Pérez Taype

Luengo, O. (2011). Debates electorales en televisión: una aproximación preliminar a sus efectos inmediatos. Revista Española de Ciencia Política, (25), 81-96. https://recyt.fecyt.es/index.php/recp/article/view/37516

Proust, V. (2018). Ironía y descortesía en los debates radiales en Chile: estrategias discursivas para ganar prestigio. Raled, 18 (2), 93-109.

Vergara, A., Tretti, V. y Herrera, A. (2020). Descortesía en los debates políticos costarricenses del 2018. Revista de Comunicación política. 2, 99-122. https:// rcp.uanl.mx/index.php/rcp/article/view/25

\section{Trayectoria académica de la autora}

Sindy Pérez Taype es bachiller en Lingüística por la Universidad Nacional Mayor de San Marcos. Actualmente, está cursando la maestría en Lingüística por la Universidad Nacional Mayor de San Marcos. Sus intereses investigativos se centran en las áreas de la lingüística cognitiva, el análisis del discurso y la pragmática. 